Schweizerische Zeitschrift für

\section{Christoph Bachmann}

\section{Einleitung}

Seit einiger Zeit gibt es in der Schweiz Johanniskraut-Präparate, die aufgrund von neueren Studien für die Indikation «leichte bis mittelgradige depressive Episoden» zugelassen sind. Eines davon ist der Extrakt STW3-VI. Jede Filmtablette enthält (gemäss ArzneimittelFachinformation) Hyperici herbae extractum ethanolicum siccum $900 \mathrm{mg}$ (3-6:1), was 0,9-2,7 mg Gesamthypericin (berechnet als Hypericin) entspricht. Damit ist STW3-VI das am höchsten dosierte Johanniskraut-Präparat der Schweiz. Die tägliche Einmaldosis von 900 mg fördert die Compliance der betroffenen Patienten und $\mathrm{Pa}$ tientinnen.

Verschiedene klinische, qualitativ einwandfreie Studien, die von ausgewiesenen Phytotherapie-Fachleuten durchgeführt wurden, zeigen Vorteile von STW3-VI gegenüber synthetischen Antidepressiva.

\section{Gastpar et al., 2006}

\section{Studie}

2006 publizierten Gastpar et al. [2] eine Vergleichsstudie mit Johanniskraut. Der Serotonin-Wiederaufnahmehemmer (SSRI) Citalopram diente als Vergleichsmedikament; die Studie wurde dreiarmig, mit einer Placebo-

\title{
Wirksamkeit eines hochdosierten Johanniskraut-Extraktes bei mittelschweren Depressionen: Studien bestätigen gleiche Wirksamkeit wie und bessere Sicherheit als Citalopram
}

In den letzten Jahren hat sich die Behandlung von Episoden leichter bis moderater Depressionen mit Extrakten aus Johanniskraut (Hypericum perforatum L.) auch in der traditionellen Medizin etabliert. Dafür steht eine Reihe unterschiedlicher Präparate zur Verfügung. Trotz intensiver Forschung konnte bisher nicht festgestellt werden, welche Inhaltsstoffe von $H$. perforatum für die antidepressive Wirkung verantwortlich sind. Es konnte jedoch gezeigt werden, dass Johanniskraut-Extrakte die Hemmung der zellulären Wiederaufnahme von Noradrenalin, Serotonin und Dopamin bewirken und auch mit den GABA-Rezeptoren interferieren [1].

Gruppe, angelegt. Das Ziel dieser Studie war, neben der vergleichbaren Wirksamkeit von STW3 gegenüber Citalopram auch die Überlegenheit des Johanniskraut-Extraktes gegenüber Placebo bei Patienten mit einer mittelschweren Depression zu dokumentieren. Weiter wurden die Sicherheit und die Verträglichkeit der Studienmedikamente untersucht.

\section{Design}

Die Studie wurde als doppelblinde, randomisierte Multizenterstudie angelegt und dauerte 6 Wochen. Probanden waren 394 rekrutierte Patienten mit einer mittelschweren Depression gemäss ICD-10 F32.1 oder F33.1. Die Probanden erhielten während 6 Wochen täglich $900 \mathrm{mg}$ STW3-VI als Einzeldosis, 20 mg Citalopram oder Placebo. 388 Probanden konnten in die ITT-Auswertung aufgenommen werden, hierunter 131 aus der Hypericumsowie 127 aus der Citalopram-Gruppe.

\section{Kasten. Angaben}

- STW3-VI ist in der Schweiz unter dem Mar kennamen Deprivita ${ }^{\circledR}$ (Permamed) im Handel und wird von der Grundversicherung erstattet.

- Es gibt Filmtabletten à 30 und à 90 .

- Tagestherapiekosten (90 Filmtabletten): CHF 0,74 (Publikumspreis)

Primäre Zielvariablen waren die Nichtunterlegenheit von STW3-VI gegenüber Citalopram und die Überlegenheit gegenüber Placebo. Als Nichtunterlegenheit wurde ein maximaler Unterschied von 3 Punkten auf der HAMD(Hamilton Depression)Skala definiert.

Sekundäre Zielparameter waren die Wirksamkeitsparameter Befindlichkeitsskala (BfS), klinischer Gesamteindruck (Clinical Global Impression (CGI)) sowie die Sicherheit und Verträglichkeit der Studienmedikamente.

Als Responder galten Patienten mit einer mindestens 50\%igen Verbesserung auf der HAMD-Skala während

\section{KARGER}

(C) 2017 S. Karger GmbH, Freiburg 
der Studiendauer oder mit einem HAMD-Wert $\leq 10$ am Studienende.

\section{Resultate}

Die Verbesserung im Verlauf der Studie auf der HAMD-Skala wird in Tabelle 1 dargestellt. Der fast identische Verlauf der Verbesserung des HAMD-Wertes für beide VerumGruppen ist sehr deutlich sichtbar, was die Nichtunterlegenheit von STW3-VI gegenüber Citalopram hochsignifikant zeigt $(\mathrm{p}<0,0001)$. Ebenso deutlich wird die Überlegenheit gegenüber Placebo von Tag 21 an sichtbar, die am Studienende eine ebenso hochsignifikante Überlegenheit zeigt $(\mathrm{p}<0,0001)$.

Die Verbesserung auf der BfS als sekundäre Zielvariable ist für die beiden Verum-Gruppen ebenso hochsignifikant $(p<0,0001)$. Ebenso verhält es sich mit dem CGI.

In der STW3-VI-Gruppe traten weniger unerwünschte Ereignisse (UE) auf als in den beiden anderen Gruppen. Die Hypericum-Einnahme führte zu weniger Therapieabbrüchen, als es für Citalopram und Placebo der Fall war. Der STW3-VI-Extrakt wurde von den Prüfärzten und den Probanden als besser verträglich als Citalopram bezeichnet ( $\mathrm{p}=$ 0,0005 Citalopram; $\mathrm{p}=$ 0,0234 Placebo).

\section{Zusammenfassung}

Damit dokumentiert diese Studie die Nichtunterlegenheit des Johanniskraut-Extraktes STW3-VI gegenüber einem SSRI sowie die bessere Verträglichkeit. Die vorliegende Studie zeigte zusätzlich noch die Überlegenheit gegenüber Placebo.

\section{Singer et al., 2011}

Bei einer Behandlung depressiver Episoden spielt die Rückfall- und Rezidivrate eine grosse Rolle. In diesem Sinne ist es sehr wichtig, darüber Erkenntnisse zu gewinnen. Das ist die
Tab. 1. Durchschnittliche Verbesserung auf der HAMD-Skala während der Behandlung

\begin{tabular}{llll}
\hline Verlauf & STW3-VI & Citalopram & Placebo \\
\hline Baseline & 21,9 & 21,8 & 22,0 \\
Tag 7 & 18,7 & 18,3 & 18,7 \\
Tag 21 & 13,5 & 13,7 & 15,3 \\
Tag 42 & 10,3 & 10,3 & 13,0 \\
Baseline - Tag 42 & 11,6 & 11,4 & 9,0 \\
\hline
\end{tabular}

Basis einer Studie von Singer et al. [3] aus dem Jahr 2011, welche die Dauer der Verbesserung sowie das Auftreten von Rückfällen und Rezidiven der Studie von Gastpar et al. [2] neu auswertete. Von der Gruppe der 154 Responder wurden nach 6 Monaten total 30 Rückfälle ermittelt. Diese verteilten sich folgendermassen: 14 von 54 (26\%) in der Citalopram-Gruppe, 8 von 54 (15\%) in der STW3-VI-Gruppe und 8 von 46 (17\%) in der Placebo-Gruppe. In Bezug auf den Schweregrad des Rückfalls konnten zwischen den Gruppen keine Unterschiede festgestellt werden. Nach 3 Jahren wurde für die 3 Gruppen die Summe der Rückfall- und Rezidivraten eruiert. Auch hier lag die STW3-VI-Gruppe mit 17 von 54 (31\%) tiefer als die CitalopramGruppe (21 von 54, 39\%) und die Placebo-Gruppe (24 von 46, 52\%).

Die ergänzende Analyse zur Studie von Gastpar et al. [2] zeigt also erneut eine Überlegenheit von STW3-VI in Bezug auf die Anzahl der Rückfälle und Rezidive.

\section{Versorgungsstudie \\ Kresimon et al., 2012}

Herkömmliche klinische Studien sind sehr wichtig, weil nur so die Wirksamkeit von Wirkstoffen und Behandlungsmethoden objektiv ermittelt werden kann. Mit ihren strengen Rahmenbedingungen schaffen solche Studien aber Bedingungen, die nicht dem Praxisalltag von Ärzten entsprechen. Deshalb hat sich in den letzten Jahren eine neue Art von Studien, sogenannte Versorgungsstudien, etabliert, mit denen Wirksamkeit, Sicherheit und Compliance im Praxisalltag erforscht werden. Damit verbunden sind Kosten-Nutzen-Analysen und Kosten-Effektivitäts-Analysen.

In diesem Sinne verglichen Kresimon et al. [4] in einer 2012 publizierten Studie die Behandlung einer mittelschweren Depression einerseits mit STW3-VI und anderseits mit beliebigen SSRI im Praxisalltag niedergelassener Ärzte in Deutschland.

In diese nichtinterventionelle prospektive Beobachtungsstudie wurden 77 Hausärzte/Neurologen/Psychiater einbezogen, welche Patienten mit entsprechenden Depressionen behandelten. Die Wirksamkeit wurde mit dem Quick Inventory of Depressive Symptomatology (QISD) bei Baseline, nach 3-4 Wochen sowie nach 3 und $6 \mathrm{Mo}-$ naten überprüft. Auch am Ende jedes Quartals wurde der Zustand der Patienten ermittelt. Weitere Prüfparameter waren die Anzahl an UE und von unerwünschten Arzneimittelwirkungen (UAW) sowie die Compliance. Die Prüfärzte bewerteten diese $\mathrm{Pa}$ rameter anhand einer fünfstufigen Skala.

Nach 6 Monaten Behandlung ergab sich folgendes Bild: Die STW3-VIGruppe zeigte gegenüber der SSRIGruppe eine vergleichbar gute Wirksamkeit, jedoch eine signifikant bessere Verträglichkeit und Compliance. Die Therapie mit STW3-VI erzeugte weniger direkte Therapiekosten und ebenso weniger indirekte Kosten sowie gleichzeitig auch ein besseres Kosten-Nutzwert-Verhältnis. Ebenso wies die Kosten-Effektivitäts-Analyse die Therapie mit STW3-VI als die kosteneffektivere Therapieart aus. 


\section{Fazit}

Die drei hier besprochenen Studien zeigen für den Johanniskraut-Extrakt STW3-VI folgende Resultate:

- STW3-VI ist sowohl in der kurzzeitigen Behandlung (6 Wochen) als auch bei Langzeitbehandlungen (24 Wochen) von Patienten mit mittelschwerer Depression gleich wirksam wie SSRI sowie signifikant wirksamer als Placebo.

- In Bezug auf die Sicherheit (UE, UAW) ist STW3-VI gegenüber SSRI deutlich überlegen.

- Die Dauer der Wirksamkeit ist länger als bei SSRI, die Rückfall- und Rezidivrate deutlich geringer und die Compliance deutlich besser.

\section{Literatur}

1 Honegger U: Johanniskraut - dem Wirkungsmechanismus einen Schritt näher. Phytotherapie 2007;7:15-17.

2 Gastpar M, et al: Comparative efficacy and safety of a once-daily dosage of Hypericum extract STW3-VI and citalopram in patients with moderate depressions: a double-blind, randomised, multicentre, placebo-controlled study. Pharmacopsychiatry 2006;39:66-75.

3 Singer A, et al: Duration of response after treatment of mild to moderate depressions with $\mathrm{Hy}$ pericum extract STW3-VI, citalopram and placebo: a reanalysis of data from a controlled clinical trial. Phytomedicine 2011;18:739-742.
- Das Kosten-Nutzen-Verhältnis für STW3-VI ist besser als für SSRI.

Damit erweist sich STW3-VI gegenüber SSRI als wirksame, sichere und kostengünstigere Alternative zur Behandlung leichter und mittelschwerer Depressionen.
4 Kresimon J, et al: Versorgung von Patienten mit mittelschwerer Depression unter Therapie mit Hypericum-Extrakt STW3-VI im Vergleich zu selektiven Serotonin-Wiederaufnahmehemmern (SSRI) im Praxisalltag. Gesundh Ökon Qual Manag 2012;DOI: 10.1055/s-00311299460. 